QUARTERLY OF APPLIED MATHEMATICS

VOLUME LXVI, NUMBER 3

SEPTEMBER 2008, PAGES 521-537

S 0033-569X(08)01090-5

Article electronically published on July 3, 2008

\title{
STOCHASTIC PARTIAL DIFFERENTIAL EQUATIONS DRIVEN BY MULTI-PARAMETER WHITE NOISE OF LÉVY PROCESSES
}

\author{
BY \\ BERNT ØKSENDAL
}

Center of Mathematics for Applications (CMA), Department of Mathematics, University of Oslo, P. O. Box 1053 Blindern, N-0316 Oslo, Norway,

Norwegian School of Economics and Business Administration (NHH), N-5014 Bergen, Norway

Abstract. We give a short introduction to the white noise theory for multiparameter Lévy processes and its application to stochastic partial differential equations driven by such processes. Examples include temperature distribution with a Lévy white noise heat source, and heat propagation with a multiplicative Lévy white noise heat source.

1. Introduction. The white noise theory was originally developed by T. Hida for Brownian motion $\{B(t)\}_{t \geq 0}$. See e.g. [7] and [8] and the references therein. The main idea was that a rigorous mathematical foundation for the time derivative of $B(t)$,

$$
\dot{B}(t)=\frac{d}{d t} B(t) \quad \text { (called white noise) }
$$

(which does not exist in the ordinary sense), would make it easier to handle stochastic calculus involving Brownian motion in general. This turned out to be a fruitful idea, both in connection with stochastic differential equations (see e.g. 9]) and MalliavinHida calculus (see e.g. the forthcoming book [6]). In particular, for stochastic partial differential equations (SPDEs) with multi-parameter noise, the white noise approach is useful because it provides solutions (in a weak sense) also when classical solutions do not exist.

In view of the success of the Brownian white noise theory, it has become natural to try to extend it to the wider family of Lévy processes. Such an extension is also of interest from the point of view of applications, because stochastic processes with jumps are useful in mathematical modelling, in e.g. physics, biology and economics. A white noise theory for Lévy processes was developed in the papers [5], [3], 14, [18] and [16].

In these papers applications were also given, e.g., to SPDEs or finance.

Received May 15, 2007.

2000 Mathematics Subject Classification. Primary 60H15, 60H40; Secondary 60G51, 60G57, 60G60, $35 \mathrm{R} 60$.

I want to thank Yaozhong $\mathrm{Hu}$ and Jiang Lun $\mathrm{Wu}$ for valuable communications.

E-mail address: oksendal@math.uio.no

(C) 2008 Brown University Reverts to public domain 28 years from publication 
The purpose of this paper is to give a short survey of this Lévy white noise theory and its applications to SPDEs. For proofs and more details we refer to the papers above, or to the exposition in Chapter 5 of 9 (Second Edition).

The outline of this paper is as follows. In Section 2 we briefly recall the basic definitions and properties of Lévy processes. In Section 3 we give a short presentation of the white noise theory for multi-parameter Lévy processes (sometimes called Lévy fields), together with a general white noise solution method for SPDEs. Finally, in Section 4 some examples are given of SPDEs solved by this method.

\section{Background on Lévy processes.}

Definition 2.1. Let $(\Omega, \mathcal{F}, P)$ be a probability space. A Lévy process on this space is a map

$$
\eta:[0, \infty) \times \Omega \rightarrow \mathbb{R}
$$

with the following properties:

(i) $\eta$ has stationary, independent increments;

(ii) $\eta$ has càdlàg paths, i.e. $t \rightarrow \eta(t)$ is right continuous with left sided limits;

(iii) $\eta$ is stochastically continuous; i.e., for all $t \geq 0, \epsilon>0$ we have

$\lim _{s \rightarrow t} P(|\eta(s)-\eta(t)|>\epsilon)=0$

(iv) $\eta(0)=0$.

The jump of $\eta$ at time $t$ is defined by

$$
\Delta \eta(t):=\eta(t)-\eta\left(t^{-}\right) .
$$

The jump measure of $\eta$ is defined by

$$
\begin{aligned}
N\left(\left(t_{1}, t_{2}\right], U\right)= & \text { the number of jumps of } \eta \text { in the time interval }\left(t_{1}, t_{2}\right] \\
& \text { and jump size } z=\Delta \eta(s) \in U ; s \in\left(t_{1}, t_{2}\right] .
\end{aligned}
$$

Here $0 \leq t_{1}<t_{2}<\infty$ and $U \in \mathcal{B}\left(\mathbb{R}_{0}\right):=$ the family of Borel sets $U$ with $\bar{U} \subset \mathbb{R}_{0}:=$ $\mathbb{R} \backslash\{0\}$.

The Lévy measure $\nu$ of $\eta(\cdot)$ is defined by

$$
\nu(U):=E[N((0,1], U)], \quad U \in \mathcal{B}\left(\mathbb{R}_{0}\right),
$$

where $E$ denotes expectation with respect to $P$. In general we have

$$
\int_{\mathbb{R}_{0}} \min \left(1, z^{2}\right) \nu(d z)<\infty
$$

(see e.g. 1], 4] or 21]) but note that we may have

$$
\int_{\mathbb{R}_{0}} \min (1,|z|) \nu(d z)=\infty .
$$

In particular, the process $t \rightarrow \eta(t)$ need not have a finite variation. Such Lévy processes are important in financial modelling. See e.g. the disucssion of infinite activity models in 4 .

From now on we will assume that

$$
\int_{\mathbb{R}_{0}} z^{2} \nu(d z)<\infty
$$


which is equivalent to assuming that

$$
\left.E\left[\eta^{2}(t)\right)\right]<\infty \text { for all } t \geq 0 .
$$

The compensated jump measure (compensated Poisson random measure) of $\eta(\cdot)$ is defined by

$$
\tilde{N}(d t, d z)=N(d t, d z)-\nu(d z) d t .
$$

The Lévy-Itô representation theorem states that there exist constants $a \in \mathbb{R}, \sigma \in \mathbb{R}$ such that

$$
\eta(t)=a t+\sigma B(t)+\int_{0}^{t} \int_{\mathbb{R}_{0}} z \tilde{N}(d s, d z)
$$

where $B(t)=B(t, \omega)$ is a Brownian motion, independent of the pure jump Lévy martingale

$$
\eta_{0}(t):=\int_{0}^{t} \int_{\mathbb{R}} z \tilde{N}(d s, d z) .
$$

In view of this we may regard Lévy processes as natural generalizations of Brownian motion to discontinuous processes. Moreover, it becomes natural to consider stochastic differential equations of the form

$$
d X(t)=b(t, X(t)) d t+\sigma(t, X(t)) d B(t)+\int_{\mathbb{R}_{0}} \gamma(t, X(t), z) \tilde{N}(d t, d z)
$$

for given functions $b: \mathbb{R} \times \mathbb{R} \rightarrow \mathbb{R}, \sigma: \mathbb{R} \times \mathbb{R} \rightarrow \mathbb{R}$ and $\gamma: \mathbb{R} \times \mathbb{R} \times \mathbb{R}_{0} \rightarrow \mathbb{R}$ satisfying certain growth conditions. See e.g. 20], Chapter 1.

In the Brownian motion case it is well known that it is possible to define the time derivative

$$
\dot{B}(t):=\frac{d}{d t} B(t)
$$

in a weak sense (distribution sense). There are 2 ways of doing this:

1) For a.a. $\omega$ the map $t \rightarrow \dot{B}(t, \omega)$ is a distribution on $\mathbb{R}$ (in the classical sense) 22.

2) $t \rightarrow \dot{B}(t)$ is a map from $[0, \infty)$ into $(\mathcal{S})^{*}$, where $(\mathcal{S})^{*}$ is a space of stochastic distributions [8, 9 .

The advantage with the second interpretation is that it applies to nonlinear equations. Indeed, we have

$$
\int_{0}^{T} \psi(t) d B(t)=\int_{0}^{T} \psi(t) \diamond \dot{B}(t) d t,
$$

where the last integral is an $(\mathcal{S})^{*}$-valued integral, and $\diamond$ denotes the Wick product in $(\mathcal{S})^{*}$ (see [2], [15] and also 9]). Moreover, the approach 2) applies to the multiparameter case, in the sense that we can define

$$
\dot{B}\left(x_{1}, \ldots, x_{k}\right)=\frac{d^{k}}{d x_{1} \ldots d x_{k}} B\left(x_{1}, \ldots, x_{k}\right) \in(\mathcal{S})^{*}
$$

( $k$-parameter Brownian white noise), where $B\left(x_{1}, \ldots, x_{k}\right)$ is $k$-parameter Brownian motion (the $k$-parameter Brownian sheet). This can be used to study SPDEs driven by the white noise $\dot{B}\left(x_{1}, \ldots, x_{k}\right)$ in the same way as in the 1 -parameter case $(k=1)$. See $\underline{9}$. 
A natural question is: Can this approach be extended to Lévy processes? Can we define the Lévy white noise

$$
\dot{\eta}(t):=\frac{d}{d t} \eta(t) \quad \text { in }(\mathcal{S})_{\eta}^{*},
$$

where $(\mathcal{S})_{\eta}^{*}$ is a corresponding space of stochastic distributions, and more generally

$$
\dot{\eta}\left(x_{1}, \ldots, x_{k}\right)=\frac{d^{k}}{\partial x_{1} \ldots \partial x_{k}} \eta\left(x_{1}, \ldots, x_{k}\right)
$$

(the $k$-parameter Lévy white noise) and apply it to study SPDEs driven by such noise?

REMARK. Why bother with singular objects like the white noises

$$
\dot{B}(t)=\frac{d}{d t} B(t) \quad \text { and } \quad \dot{\eta}(t)=\frac{d}{d t} \eta(t) ?
$$

Why not use smoothed versions instead? We answer this by considering a simple example.

EXAMPLE 2.2. Let $B_{n}(t)$ be a smooth approximation to $B(t)$. Then the equation

$$
d X_{n}(t)=\mu X_{n}(t) d t+\sigma X_{n} \frac{d B_{n}}{d t} d t, \quad X_{n}(0)=x>0
$$

has the solution

$$
X_{n}(t)=x \exp \left(\mu t+\sigma B_{n}(t)\right) \quad(\mu, \sigma \neq 0 \text { constants }) .
$$

On the other hand, the "singular white noise equation" (Itô equation)

$$
d X(t)=\mu X(t) d t+\sigma X(t) d B(t), \quad X(0)=x>0
$$

has the solution

$$
X(t)=x \exp \left(\left(\mu-\frac{1}{2} \sigma^{2}\right) t+\sigma B(t)\right) .
$$

Note that $\lim _{n \rightarrow \infty} X_{n}(t) \neq X(t)$ even though $\lim _{n \rightarrow \infty} B_{n}(t)=B(t)$.

Thus we see that smoothing the noise gives a totally different equation!

Here are some examples of stochastic partial differential equations which are solvable by the method discussed in this paper:

EXAMPLE 2.3. Temperature distribution in a region with a Lévy white noise heat source $\dot{\eta}(x)$ :

$$
\left\{\begin{array}{l}
\Delta U(x)=-\dot{\eta}(x) ; \quad x \in D \\
U(x)=0 ; \quad x \in \partial D
\end{array}\right.
$$

where $D$ is a given domain in $\mathbb{R}^{d}$ and $\Delta=\sum_{i=1}^{d} \frac{\partial^{2}}{\partial x_{i}^{2}}$ is the Laplacian operator.

EXAMPLE 2.4. Waves in a medium subject to a Lévy white noise force:

$$
\begin{cases}\frac{\partial^{2} U}{\partial t^{2}}(t, x)-\Delta U(t, x)=F(t, x) ; & (t, x) \in[0, \infty) \times \mathbb{R}^{m} \\ U(0, x)=G(x) ; & x \in \mathbb{R}^{m} \\ \frac{\partial U}{\partial t}(0, x)=H(x) ; & x \in \mathbb{R}^{m}\end{cases}
$$

where $F, G$ and $H$ are Lévy white noise functionals, e.g. space-time or space white noise.

How do we solve such SPDEs? 
EXAMPLE 2.5. Heat propagation in a domain with a (multiplicative) Lévy white noise potential $\dot{\eta}(t, x)$ :

$$
\frac{\partial U}{\partial t}(t, x)=\Delta U(t, x)+{ }^{\prime \prime} U(t, x) \cdot \dot{\eta}(t, x)^{\prime \prime} .
$$

How do we interpret this equation rigorously? How do we solve it?

We will use white noise theory to answer these questions.

3. White noise theory for a Lévy field. We now give a brief review of the white noise theory for a $d$-parameter Lévy process. For details and proofs we refer to [9] (Second Edition) and the references therein.

Let $\nu$ be a given measure on $\mathcal{B}_{0}\left(\mathbb{R}_{0}\right)$ such that

$$
M:=\int_{\mathbb{R}} z^{2} \nu(d z)<\infty .
$$

We will construct a $d$-parameter Lévy process $\eta(x) ; x=\left(x_{1}, \ldots, x_{d}\right) \in \mathbb{R}^{d}$, such that $\nu$ is the Lévy measure of $\eta(\cdot)$, in the sense that

$$
\nu(F)=E[N(1,1, \ldots, 1 ; F)],
$$

where $N(x ; F)=N(x, F, \omega): \mathbb{R}^{d} \times \mathcal{B}_{+}\left(\mathbb{R}_{0}\right) \times \Omega \rightarrow \mathbb{R}$ is the jump measure of $\eta(\cdot)$, defined by

$$
\begin{aligned}
N\left(x_{1}, x_{2}, \ldots, x_{d} ; F\right) & =\text { the number of jumps } \Delta \eta(u)=\eta(u)-\eta\left(u^{-}\right) \text {of size } \\
\Delta \eta(u) & \in F \text { when } u_{i} \leq x_{i} ; 1 \leq i \leq n, u=\left(u_{1}, \ldots, u_{d}\right) \in \mathbb{R}^{d} .
\end{aligned}
$$

Let $\mathcal{S}\left(\mathbb{R}^{d}\right)$ denote the Schwartz space of rapidly decreasing smooth functions on $\mathbb{R}^{d}$ and let $\Omega=\mathcal{S}^{\prime}\left(\mathbb{R}^{d}\right)$ be its dual, called the space of tempered distributions.

DeFinition 3.1. The $d$-parameter Lévy white noise probability measure is the measure $P=P^{(L)}$ defined on the Borel $\sigma$-algebra $\mathcal{B}(\Omega)$ of subsets of $\Omega$ by

$$
\int_{\Omega} e^{i\langle\omega, f\rangle} d P(\omega)=\exp \left(\int_{\mathbb{R}^{d}} \psi(f(y)) d y\right), \quad f \in \mathcal{S}\left(\mathbb{R}^{d}\right),
$$

where

$$
\psi(u)=\int_{\mathbb{R}}\left(e^{i u \cdot z}-1-i u \cdot z\right) \nu(d z)
$$

and $\langle\omega, f\rangle=\omega(f)$ denotes the action of $\omega \in \mathcal{S}^{\prime}\left(\mathbb{R}^{d}\right)$ on $f \in \mathcal{S}\left(\mathbb{R}^{d}\right)$.

The triple $\left(\Omega ; \mathcal{B}(\Omega), P^{(L)}\right)$ is called the (d-parameter) Lévy white noise probability space.

For simplicity of notation we write $P=P^{(L)}$ from now on.

Remark. The existence of $P$ follows from the Bochner-Minlos theorem: The map

$$
F: f \rightarrow \exp \left(\int_{\mathbb{R}^{d}} \psi(f(y)) d y\right), \quad f \in \mathcal{S}\left(\mathbb{R}^{d}\right)
$$

is positive definite on $\mathcal{S}\left(\mathbb{R}^{d}\right)$, i.e.

$$
\sum_{i=1}^{m} z_{j} \bar{z}_{k} F\left(f_{j}-f_{k}\right) \geq 0 \quad \text { for all } z_{j} \in \mathbb{C}, f_{j} \in \mathcal{S}\left(\mathbb{R}^{d}\right), 1 \leq j \leq m . m=1,2, \ldots
$$

Lemma 3.2. Let $g \in \mathcal{S}\left(\mathbb{R}^{d}\right)$ and put $M:=\int_{\mathbb{R}} z^{2} \nu(d z)<\infty$. Then, with $E=E_{P}$, 
(i) $E[\langle\cdot, g\rangle]=0$,

(ii) $\operatorname{Var}_{P}[\langle\cdot, g\rangle]=E\left[\langle\cdot, g\rangle^{2}\right]=M \int_{\mathbb{R}^{d}} g^{2}(y) d y$.

Using this we can extend the definition of $\langle\omega, f\rangle$ from $f \in \mathcal{S}\left(\mathbb{R}^{d}\right)$ to $f \in L^{2}\left(\mathbb{R}^{d}\right)$ as follows:

If $f \in L^{2}\left(\mathbb{R}^{d}\right)$ choose $f_{n} \in \mathcal{S}\left(\mathbb{R}^{d}\right)$ s.t. $f_{n} \rightarrow f$ in $L^{2}\left(\mathbb{R}^{d}\right)$. Then the limit

$$
\lim _{n \rightarrow \infty}\left\langle\omega, f_{n}\right\rangle
$$

exists in $L^{2}(P)$ and is independent of the sequence chosen. This limit is denoted by $\langle\omega, f\rangle$.

Theorem 3.3. For $x=\left(x_{1}, \ldots, x_{d}\right) \in \mathbb{R}^{d}$ define

$$
\tilde{\eta}(x)=\tilde{\eta}\left(x_{1}, \ldots, x_{d}\right)=\left\langle\omega, \chi_{[0, x]}(\cdot)\right\rangle,
$$

where

$$
\chi_{[0, x]}(y)=\chi_{\left[0, x_{1}\right]}\left(y_{1}\right) \ldots \chi_{\left[0, x_{d}\right]}\left(y_{d}\right), \quad y=\left(y_{1}, \ldots, d_{d}\right) \in \mathbb{R}^{d}
$$

with

$$
\chi_{\left[0, x_{i}\right]}\left(y_{i}\right)= \begin{cases}1 & \text { if } 0 \leq y_{i} \leq x_{i} \text { or } x_{i} \leq y_{i} \leq 0 \\ 0 & \text { otherwise. }\end{cases}
$$

Then $\tilde{\eta}(x)$ has the following properties:

(i) $\tilde{\eta}(x)=0$ if one of the components of $x$ is 0 ;

(ii) $\tilde{\eta}$ has independent increments;

(iii) $\tilde{\eta}$ has stationary increments;

(iv) $\tilde{\eta}(\cdot)$ has a càdlàg version, denoted by $\eta(\cdot)$.

This version $\eta(x), x \in \mathbb{R}^{d}$, is the pure jump Lévy field that we will work with from now on.

REMARK. If $d=1$, then this process $\eta(t)$ coincides with the classical Lévy process with the given Lévy measure $\nu$.

By our choice (3.5) of the function $\psi$ it follows by the Lévy-Khintchine formula that $\eta(x)$ is a pure jump Lévy martingale of the form

$$
\eta(x)=\int_{0}^{x} \int_{\mathbb{R}} z \tilde{N}(d y, d z),
$$

where $\tilde{N}(d y, d z)=N(d y, d z)-\nu(d z) d y$ and, by definition,

$$
\int_{0}^{x} f(y) d y=\int_{0}^{x_{n}} \int_{0}^{x_{n-1}} \cdots \int_{0}^{x_{1}} f(y) d y_{1} \ldots d y_{n}
$$

if $x=\left(x_{1}, \ldots, x_{n}\right)$. We may regard $\eta(x)=\eta(x, \omega)$ as a random measure $\eta=\eta(\omega)$ applied to the rectangle $\left[0, x_{1}\right] \times \cdots \times\left[0, x_{n}\right]$. As such it extends in a natural way to a random measure on the Borel subsets of $\mathbb{R}^{d}$. See 10 .

If $f=f\left(x^{(1)}, z_{1}, \ldots, x^{(n)}, z_{n}\right):\left(\mathbb{R}^{d} \times \mathbb{R}_{0}\right)^{n} \rightarrow \mathbb{R}$ we define the symmetrization $\hat{f}$ of $f$ as the symmetrization with respect to the $n$ variables $y_{1}=\left(x^{(1)}, z_{1}\right), y_{2}=$ $\left(x^{(2)}, z_{2}\right), \ldots, y_{n}=\left(x^{(n)}, z_{n}\right)$, i.e.

$$
\hat{f}\left(y_{1}, \ldots, y_{n}\right)=\frac{1}{n !} \sum_{\sigma} f\left(y_{\sigma_{1}}, \ldots, y_{\sigma_{n}}\right),
$$


the sum being taken over all permutations $\sigma$ of $(1,2, \ldots, n)$. We let $\hat{L}^{2}\left((\lambda \times \nu)^{n}\right)$ denote the set of all symmetric functions $f \in L^{2}\left((\lambda \times \nu)^{n}\right)$, where $\lambda$ denotes Lebesgue measure on $\mathbb{R}^{d}$. For $f \in \hat{L}^{2}\left((\lambda \times \nu)^{n}\right)$ we define

$$
I_{n}(f)=n ! \int_{G_{n}} f\left(x^{(1)}, z_{1}, \ldots, x^{(n)}, z_{n}\right) \tilde{N}\left(d x^{(1)}, d z_{1}\right) \ldots \tilde{N}\left(d x^{(n)}, d z_{n}\right),
$$

where

$$
G_{n}=\left\{\left(x^{(1)}, z_{1}, \ldots, x^{(n)}, z_{n}\right) \in\left(\mathbb{R}^{d} \times \mathbb{R}\right)^{n} ; x_{j}^{(1)} \leq x_{j}^{(2)} \leq \cdots \leq x_{j}^{(n)} \text { for all } j=1, \ldots, d\right\} .
$$

Theorem 3.4 (Chaos expansion I). (i) Every $F \in L^{2}(P)$ has a unique representation

$$
F=\sum_{n=0}^{\infty} I_{n}(f) \quad \text { with } f_{n} \in \hat{L}^{2}\left((\lambda \times \nu)^{n}\right), I_{0}\left(f_{0}\right)=E[F] .
$$

(ii) Moreover, we have the isometry

$$
\|F\|_{L^{2}(P)}^{2}=\sum_{n=0}^{\infty} n !\left\|f_{n}\right\|_{L^{2}\left((\lambda \times \nu)^{n}\right)}^{2} .
$$

EXAMPLE 3.5. $F=\eta(x)$ has the expansion

$$
\eta(x)=\int_{0}^{x} \int_{\mathbb{R}} z \tilde{N}(d y, d z)=I_{1}\left(f_{1}\right),
$$

with

$$
f_{1}(y, z)=\chi_{\left[0 . x_{1}\right]}\left(y_{1}\right) \ldots \chi_{\left[0, x_{d}\right]}\left(y_{d}\right) z .
$$

Definition 3.6 (Skorohod integrals). ( $d=1$ : Kabanov 1974 [1], 12]) Let $Y(x)=$ $\sum_{n=0}^{\infty} I_{n}\left(f_{n}(\cdot, x)\right),(x, \omega) \in \mathbb{R}^{d} \times \Omega$, be a random field, with the property that

$$
\sum_{n=0}^{\infty}(n+1) !\left\|\tilde{f}_{n}\right\|_{L^{2}\left((\lambda \times \nu)^{n+1}\right)}^{2}<\infty,
$$

where $\tilde{f}_{n}=\tilde{f}_{n}\left(x^{(1)}, z_{1}, \ldots, x^{(n)}, z_{n}, x, z\right)$ is the symmetrization of $h_{n}:=z f_{n}$ with respect to the $n+1$ variables $y_{1}=\left(x^{(1)}, z_{1}\right), \ldots, y_{n}=\left(x^{(n)}, z_{n}\right), y_{n+1}=(x, z)=:\left(x^{(n+1)}, z_{n+1}\right)$.

Then the Skorohod integral of $Y(\cdot)$ with respect to $\eta(\cdot)$ is defined by

$$
\int_{\mathbb{R}^{d}} Y(x) \delta \eta(x)=\sum_{n=0}^{\infty} I_{n+1}\left(\tilde{f}_{n}\right) .
$$

If $Y(\cdot)$ is adapted, in the sense that for all $x$ the random variable $Y(x)$ is measurable w.r.t. the $\sigma$-algebra $\mathcal{F}_{x}$ generated by

$$
\left\{\eta(y) ; y_{1} \leq x_{1}, \ldots, y_{d} \leq x_{d}\right\}
$$

and $E\left[\int_{\mathbb{R}^{d}} Y^{2}(x) d x\right]<\infty$, then

$$
\int Y(x) \delta \eta(x)=\int Y(x) d \eta(x) \quad(=\text { the Itô integral [10] }) .
$$


Assume from now on that the Lévy measure $\nu$ satisfies the following integrability condition:

For all $\epsilon>0$ there exists $\lambda>0$ such that

$$
\int_{|z| \geq \epsilon} \exp (\lambda|z|) \nu(d z)<\infty .
$$

This condition implies that $\nu$ has finite moments of order $n$ for all $n \geq 2$. It is trivially satisfied if $\nu$ is supported on $[-R, R]$ for some $R>0$.

The condition (3.12) implies that the polynomials are dense in $L^{2}(\rho)$, where

$$
d \rho(z):=z^{2}(\nu(d z)
$$

(see [17).

Now let $\left\{\ell_{m}\right\}_{m=0}^{\infty}=\left\{1, \ell_{1}, \ell_{2}, \ldots\right\}$ be the orthogonalization of $\left\{1, z, z^{2}, \ldots\right\}$ with respect to the inner product of $L^{2}(\rho)$. Define

$$
p_{j}(z):=\left\|\ell_{j-1}\right\|_{L^{2}(\rho)}^{-1} z \ell_{j-1}(z), \quad j=1,2, \ldots
$$

Then $\left\{p_{j}(z)\right\}_{j=1}^{\infty}$ is an orthonormal basis for $L^{2}(\nu)$. Note that $p_{1}(z)=m_{2}^{-1} z$, or $z=$ $m_{2} p_{1}(z)$, where $m_{2}=\left(\int_{\mathbb{R}_{0}} z^{2} \nu(d z)\right)^{1 / 2}$.

Let $\left\{\xi_{i}(t)\right\}_{i=1}^{\infty}$ be the Hermite functions on $\mathbb{R}$. For $\gamma=\left(\gamma_{1}, \ldots, \gamma_{d}\right) \in \mathbb{N}^{d}$ define

$$
\xi_{\gamma}\left(x_{1}, \ldots, x_{d}\right)=\xi_{\gamma_{1}}\left(x_{1}\right) \xi_{\gamma_{2}}\left(x_{2}\right) \ldots \xi_{\gamma_{d}}\left(x_{d}\right),
$$

i.e.,

$$
\xi_{\gamma}=\xi_{\gamma_{1}} \otimes \xi_{\gamma_{2}} \otimes \cdots \otimes \xi_{\gamma_{d}} .
$$

Then $\left\{\xi_{\gamma}\right\}_{\gamma \in \mathbb{N}^{d}}$ is an orthonormal basis for $L^{2}\left(\mathbb{R}^{d}\right)$. We may assume that $\mathbb{N}^{d}$ is ordered, $\mathbb{N}^{d}=\left\{\gamma^{(1)}, \gamma^{(2)}, \ldots\right\}$, such that

$$
i<j \Rightarrow \gamma_{1}^{(i)}+\gamma_{2}^{(i)}+\cdots+\gamma_{d}^{(i)} \leq \gamma_{1}^{(j)}+\gamma_{2}^{(j)}+\cdots+\gamma_{d}^{(j)} .
$$

To simplify the notation we write from now on

$$
\xi_{i}(x):=\xi_{\gamma^{(i)}}(x), \quad i=1,2, \ldots, \quad x \in \mathbb{R}^{d} .
$$

Define the bijective map

$$
\kappa: \mathbb{N} \times \mathbb{N} \rightarrow \mathbb{N}
$$

by

$$
\kappa(i, j)=j+(i+j-2)(i+j-1) / 2 .
$$

Let $\left\{\xi_{i}(x)\right\}_{i=1}^{\infty}$ be the tensor products above. Then if $k=\kappa(i, j)$ we define

$$
\delta_{k}(x, z)=\delta_{\kappa(i, j)}(x, z)=\xi_{i}(x) p_{j}(z), \quad(i, j) \in \mathbb{N} \times \mathbb{N} .
$$

Let $\mathcal{J}$ be the set of all multi-indices $\alpha=\left(\alpha_{1}, \alpha_{2}, \ldots, \alpha_{m}\right)$ where $\alpha_{i} \in \mathbb{N} \cup\{0\}, m=1,2, \ldots$ We put

$$
\text { Index } \alpha=\max \left\{j ; \alpha_{j} \neq 0\right\}
$$

and

$$
|\alpha|=\alpha_{1}+\alpha_{2}+\cdots+\alpha_{j}
$$


For $\alpha \in \mathcal{J}$ with Index $\alpha=j$ and $|\alpha|=m$ we define the function $\delta^{\otimes \alpha}$ by

$$
\begin{aligned}
& \delta^{\otimes \alpha}\left(x^{(1)}, z_{1}, \ldots, x^{(m)}, z_{m}\right)=\delta_{1}^{\otimes \alpha_{1}} \otimes \cdots \otimes \delta_{j}^{\otimes \alpha_{j}}\left(x^{(1)}, z_{1}, \ldots, x^{(m)}, z_{m}\right) \\
& =\underbrace{\delta_{1}\left(x^{(1)}, z_{1}\right) \cdots \delta_{1}\left(x^{\left(\alpha_{1}\right)}, z_{\alpha_{1}}\right)}_{\alpha_{1} \text { factors }} \cdots \underbrace{\delta_{j}\left(x^{\left(m-\alpha_{j}+1\right)}, z_{m-\alpha_{j}+1}\right) \cdots \delta_{j}\left(x^{(m)}, z_{m}\right)}_{\alpha_{j} \text { factors }}
\end{aligned}
$$

(we set $\delta_{i}^{\otimes 0}=1$ ).

Finally we define the symmetrized tensor product of the $\delta_{k}$ 's, denoted by $\delta^{\hat{\otimes} \alpha}$, as follows:

$$
\begin{aligned}
& \delta^{\hat{\otimes} \alpha}\left(x^{(1)}, z_{1}, \ldots, x^{(m)}, z_{m}\right)=\left(\widehat{\delta^{\otimes \alpha}}\right)\left(x^{(1)}, z_{1}, \ldots, x^{(m)}, z_{m}\right) \\
& \quad=\delta_{1}^{\hat{\otimes} \alpha} \hat{\otimes} \cdots \hat{\otimes} \delta_{j}^{\hat{\otimes} \alpha_{j}}\left(x^{(1)}, z_{1}, \ldots, x^{(m)}, z_{m}\right),
\end{aligned}
$$

where ^ denotes symmetrization.

For $\alpha \in \mathcal{J}$ define

$$
K_{\alpha}=K_{\alpha}(\omega)=I_{|\alpha|}\left(\delta^{\hat{\otimes} \alpha}\right)(\omega), \quad \omega \in \Omega,
$$

where $I_{|\alpha|}$ is the iterated integral of order $m=|\alpha|$ with respect to $\tilde{N}(\cdot, \cdot)$ :

$$
\begin{aligned}
& I_{m}\left(f\left(x^{(1)}, z_{1}, \ldots, x^{(m)}, z_{m}\right)\right) \\
& \quad=m ! \int_{G_{m}} f\left(x^{(1)}, z_{1}, \ldots, x^{(m)}, z_{m}\right) \tilde{N}\left(d x^{(1)}, d z_{1}\right) \ldots \tilde{N}\left(d x^{(m)}, d z_{m}\right),
\end{aligned}
$$

where

$$
\begin{aligned}
& G_{m}=\left\{\left(x^{(1)}, z_{1}, \ldots, x^{(m)}, z_{m}\right) \in\left(\mathbb{R}^{d} \times \mathbb{R}\right)^{m}\right. \\
& \left.\qquad x_{j}^{(1)} \leq x_{j}^{(2)} \leq \cdots \leq x_{j}^{(m)} \text { for all } j=1, \ldots, d\right\} .
\end{aligned}
$$

From now on we use the notation $\epsilon^{(k)}=(0,0, \ldots, 1)$, with 1 in the $k$ th place.

EXAMPLE 3.7.

$$
\begin{aligned}
K_{\epsilon^{(\kappa(i, j))}} & =K(0,0, \ldots, \quad \stackrel{\kappa(i, j) \text { place }}{1})=I_{1}\left(\delta^{\hat{\otimes} \epsilon^{(\kappa(i, j))}}\right) \\
& =I_{1}\left(\delta_{\kappa(i, j)}\right)=I_{1}\left(\xi_{i}(x) p_{j}(z)\right) \\
& =\int_{\mathbb{R}^{d}} \int_{\mathbb{R}} \xi_{i}(x) p_{j}(z) \tilde{N}(d x, d z) .
\end{aligned}
$$

Let

$$
f_{n}=\sum_{|\alpha|=n} a_{\alpha} \delta^{\hat{\otimes} \alpha} \in \hat{L}^{2}\left((\lambda \times \nu)^{n}\right)
$$

and

$$
g_{m}=\sum_{|\beta|=m} a_{\beta} \delta^{\hat{\otimes} \beta} \in \hat{L}^{2}\left((\lambda \times \nu)^{m}\right) .
$$

Then

$$
f_{n} \hat{\otimes} g_{m}=\sum_{|\alpha|=n} \sum_{|\beta|=m} a_{\alpha} b_{\beta} \delta^{\hat{\otimes}(\alpha+\beta)}=\sum_{|\gamma|=n+m}\left(\sum_{\alpha+\beta=\gamma} a_{\alpha} b_{\beta}\right) \delta^{\hat{\otimes} \gamma}
$$

and

$$
I_{n}\left(f_{n}\right)=\sum_{|\alpha|=n} a_{\alpha} I_{n}\left(\delta^{\hat{\otimes} \alpha)}\right)=\sum_{|\alpha|=n} a_{\alpha} K_{\alpha}
$$


and

$$
I_{n+m}\left(f_{n} \hat{\otimes} g_{m}\right)=\sum_{|\gamma|=n+m}\left(\sum_{\alpha+\beta=\gamma} a_{\alpha} b_{\beta} K_{\alpha+\beta}\right) .
$$

Combining (3.20) with (3.15) - 3.16) we get the following alternative chaos expansion:

Theorem 3.8 (Chaos expansion II). The family $\left\{K_{\alpha}\right\}_{\alpha \in \mathcal{J}}$ constitutes an orthogonal basis for $L^{2}(P)$. Thus, every $F \in L^{2}(P)$ has a unique representation

$$
F=\sum_{\alpha \in \mathcal{J}} c_{\alpha} K_{\alpha}
$$

where $c_{\alpha} \in \mathbb{R}$ for all $\alpha \in \mathcal{J}$. Moreover, we have the isometry

$$
\|F\|_{L^{2}(P)}^{2}=\sum_{\alpha \in \mathcal{J}} \alpha ! c_{\alpha}^{2}
$$

where $\alpha !=\alpha_{1} ! \ldots \alpha_{m} !$ if $\alpha=\left(\alpha_{1}, \ldots, \alpha_{m}\right) \in \mathcal{J}$.

Example 3.9. Choose $F=\eta(x)=\int_{0}^{x} \int_{\mathbb{R}} z \tilde{N}(d y, d z)$. Then

$$
\begin{aligned}
F & =\int_{\mathbb{R}^{d}} \int_{\mathbb{R}_{i=1}} \sum_{i=1}^{\infty}\left(\chi_{[0, x]}(\cdot), \xi_{i}\right)_{L^{2}(\lambda)} \xi_{i}(x) z \tilde{N}(d x, d z) \\
& =\sum_{i=1}^{\infty} \int_{0}^{x_{d}} \cdots \int_{0}^{x_{1}} \xi_{i}(y) d y_{1} \ldots d y_{\alpha}\left(\int_{\mathbb{R}^{d}} \int_{\mathbb{R}} \xi_{i}(x) z \tilde{N}(d x, d z)\right) \\
& =m_{2} \sum_{i=1}^{\infty} \int_{0}^{x_{d}} \cdots \int_{0}^{x_{1}} \xi_{i}(y) d y_{1} \ldots d y_{d} \cdot K_{\epsilon^{\kappa(i, 1)}} .
\end{aligned}
$$

Definition 3.10 (The Hida/Kondratiev spaces for Lévy fields).

\section{(i) The stochastic test function spaces.}

Let $0 \leq \rho \leq 1$. For an expansion $F=\sum_{\alpha \in \mathcal{J}} c_{\alpha} K_{\alpha} \in L^{2}(P)$ define the norm

$$
\|F\|_{\rho, k}^{2}:=\sum_{\alpha \in \mathcal{J}}(\alpha !)^{1+\rho} c_{\alpha}^{2}(2 \mathbb{N})^{k \alpha}, \quad k \in \mathbb{N} \cup\{0\},
$$

where

$$
(2 \mathbb{N})^{k \alpha}:=(2 \cdot 1)^{k \alpha_{1}}(2 \cdot 2)^{k \alpha_{2}} \cdots(2 m)^{k \alpha_{m}} \quad \text { if } \alpha=\left(\alpha_{1}, \ldots, \alpha_{m}\right) .
$$

Let

$$
(\mathcal{S})_{\rho, k}:=\left\{F \in L^{2}(P) ;\|F\|_{\rho, k}<\infty\right\}
$$

and define

$$
(\mathcal{S})_{\rho}:=\bigcap_{k=0}^{\infty}(\mathcal{S})_{\rho, k}, \quad \text { with projective topology. }
$$

(ii) The stochastic distribution spaces.

Let $0 \leq \rho \leq 1$. For an expansion $G=\sum_{\alpha \in \mathcal{J}} b_{\alpha} K_{\alpha}$ define the norm

$$
\|G\|_{-\rho,-k}^{2}:=\sum_{\alpha \in \mathcal{J}}(\alpha !)^{1-\rho} b_{\alpha}^{2}(2 \mathbb{N})^{-k \alpha}, \quad k \in \mathbb{N} \cup\{0\} .
$$

Let

$$
(\mathcal{S})_{-\rho,-k}:=\left\{G ;\|G\|_{-\rho,-k}<\infty\right\}
$$


and define

$$
(\mathcal{S})_{-\rho}:=\bigcap_{k=0}^{\infty}(\mathcal{S})_{-\rho,-k}, \quad \text { with the inductive topology. }
$$

We can regard $(\mathcal{S})_{-\rho}$ as the dual of $(\mathcal{S})_{\rho}$, by the action

$$
(F, G)=\sum_{\alpha \in \mathcal{J}} b_{\alpha} c_{\alpha} \alpha !
$$

Note that for general $0 \leq \rho \leq 1$ we have

$$
(\mathcal{S})_{1} \subset(\mathcal{S})_{\rho} \subset(\mathcal{S})_{0} \subset L^{2}(P) \subset(\mathcal{S})_{-0} \subset(\mathcal{S})_{-\rho} \subset(\mathcal{S})_{-1}
$$

The spaces $(\mathcal{S}):=(\mathcal{S})_{0}$ and $(\mathcal{S})^{*}:=(\mathcal{S})_{-0}$ are the Lévy versions of the Hida test function space and the Hida stochastic distribution space, respectively. For arbitrary $\rho \in[0,1]$ the spaces are called Kondratiev spaces.

EXAMPle 3.11. The d-parameter Lévy white noise $\dot{\eta}(x)$ of the Lévy process $\eta(x)$ is defined by the expansion

$$
\dot{\eta}(x)=m_{2} \sum_{i=1}^{\infty} \xi_{i}(x) K_{\epsilon^{\kappa(i, 1)}} .
$$

It is easy to see that

$$
\dot{\eta}(x)=\frac{\partial^{d}}{\partial x_{1} \ldots \partial x_{d}} \eta(x) \quad \text { in }(\mathcal{S})^{*} .
$$

This justifies the name Lévy white noise for $\dot{\eta}$.

Definition 3.12. The Lévy Wick product $F \diamond G$ of two elements

$$
F=\sum_{\alpha \in \mathcal{J}} a_{\alpha} K_{\alpha} \in(\mathcal{S})_{-1}, \quad G=\sum_{\beta \in \mathcal{J}} b_{\beta} K_{\beta} \in(\mathcal{S})_{-1}
$$

is defined by

$$
F \diamond G=\sum_{\alpha, \beta \in \mathcal{J}} a_{\alpha} b_{\beta} K_{\alpha+\beta}=\sum_{\gamma \in \mathcal{J}}\left(\sum_{\alpha+\beta=\gamma} a_{\alpha} b_{\beta}\right) K_{\gamma} .
$$

One can prove that all the spaces $(\mathcal{S})_{\rho},(\mathcal{S})_{-\rho}$ are closed under Wick multiplication.

EXAMPle 3.13. By (3.16) we have

$$
\begin{gathered}
K_{\epsilon^{(\kappa(i, 1))}} \diamond K_{\epsilon^{(\kappa(i, 1))}}=K_{2 \epsilon^{(\kappa(i, 1))}}=K_{(0,0, \ldots, 2)} \\
=I_{2}\left(\delta_{\kappa(i, 1)}^{\hat{\otimes} 2}\right)=I_{2}\left(\left(\xi_{i}(x) z\right)^{\hat{\otimes} 2}\right) m_{2}^{-2} .
\end{gathered}
$$

More generally, by (3.23) we see that

$$
\begin{aligned}
& I_{n}\left(f_{n}\right) \diamond I_{m}\left(g_{m}\right)=\sum_{|\alpha|=n} a_{\alpha} K_{\alpha} \diamond \sum_{|\beta|=m} b_{\beta} K_{\beta} \\
& \quad=\sum_{|\gamma|=n+m}\left(\sum_{\alpha+\beta=\gamma} a_{\alpha} b_{\beta}\right) K_{\alpha+\beta}=I_{n+m}\left(f_{n} \hat{\otimes} g_{m}\right),
\end{aligned}
$$

for $f_{n} \in \hat{L}^{2}\left((\lambda \times \nu)^{n}\right), g_{m} \in \hat{L}^{2}\left((\lambda \times \nu)^{m}\right)$.

One reason for the importance of the Wick product is the following result:

Theorem 3.14 ([2], [15]). Suppose $Y(t)$ is Skorohod integrable, $d=1$. Then

$$
\int_{\mathbb{R}} Y(t) \delta \eta(t)=\int_{\mathbb{R}} Y(t) \diamond \dot{\eta}(t) d t .
$$


REMARK. For $d>1$ the relation between the Skorohod integral and the Wick product is more complicated than (3.39). See [9].

The Hermite transform gives a relation between elements of $(\mathcal{S})_{-1}$ and analytic functions of several complex variables:

Definition 3.15. Let $F=\sum_{\alpha \in \mathcal{J}} a_{\alpha} K_{\alpha} \in(\mathcal{S})_{-1}$. Then the (Lévy) Hermite transform of $F$, denoted by $\mathcal{H} F(\zeta)$ or $\tilde{F}(\zeta)$, is defined by

$$
\mathcal{H} F\left(\zeta_{1}, \zeta_{2}, \ldots\right)=\sum_{\alpha \in \mathcal{J}} a_{\alpha} \zeta^{\alpha} \in \mathbb{C},
$$

where $\zeta=\left(\zeta_{1}, \zeta_{2}, \ldots\right) \in\left(\mathbb{C}^{\mathbb{N}}\right)_{c}$ (the set of all finite sequences of complex numbers) and

$$
\zeta^{\alpha}=\zeta_{1}^{\alpha_{1}} \cdot \zeta_{2}^{\alpha_{2}} \cdots \zeta_{m}^{\alpha_{m}} \quad \text { if } \quad \alpha=\left(\alpha_{1}, \ldots, \alpha_{m}\right) .
$$

EXAMPLE 3.16. Let $\dot{\eta}(x)=m_{2} \sum_{j=1}^{\infty} \xi_{j}(x) K_{\epsilon^{\kappa(j, 1))}}$. Then

$$
\mathcal{H}(\dot{\eta}(x))(\zeta)=m_{2} \sum_{j=1}^{\infty} \xi_{j}(x) \zeta^{\epsilon^{(\kappa(j, 1))}}=m_{2} \sum_{j=1}^{\infty} \xi_{j}(x) \zeta_{\kappa(j, 1)} .
$$

Lemma 3.17. If $F, G \in(\mathcal{S})_{-1}$, then

$$
\mathcal{H}(F \diamond G)(\zeta)=\mathcal{H}(F)(\zeta) \cdot \mathcal{H}(G)(\zeta), \quad \zeta \in\left(\mathbb{C}^{\mathbb{N}}\right)_{c} .
$$

Define for $0<R, q<\infty$ the infinite-dimensional neighborhood $N_{q}(R)$ in $\mathbb{C}^{\mathbb{N}}$ by

$$
N_{q}(R)=\left\{\left(\zeta_{1}, \zeta_{2}, \ldots\right) \in \mathbb{C}^{\mathbb{N}} ; \sum_{\alpha \neq 0}\left|\zeta^{\alpha}\right|^{2}(2 \mathbb{N})^{q \alpha}<R^{2}\right\} .
$$

Then we have the following characterization theorem:

Theorem 3.18 (9], [14]). (i) If $F=\sum_{\alpha \in \mathcal{J}} a_{\alpha} K_{\alpha} \in(\mathcal{S})_{-1}$, then there exist $q, M_{q}<\infty$ such that

$$
|\mathcal{H} F(\zeta)| \leq \sum_{\alpha \in \mathcal{J}}\left|a_{\alpha}\right|\left|\zeta^{\alpha}\right| \leq M_{q}\left(\sum_{\alpha \in \mathcal{J}}(2 \mathbb{N})^{q \alpha}\left|\zeta^{\alpha}\right|^{2}\right)^{1 / 2}
$$

for all $\zeta \in\left(\mathbb{C}^{\mathbb{N}}\right)_{c}$.

In particular, $\mathcal{H} F$ is a bounded analytic function on $N_{q}(R)$ for all $R<\infty$.

(ii) Conversely, assume that $g(\zeta):=\sum_{\alpha \in \mathcal{J}} b_{\alpha} \zeta^{\alpha}$ is a power series of $\zeta \in\left(\mathbb{C}^{\mathbb{N}}\right)_{c}$ such that there exist $q<\infty, \delta>0$ with $g(\zeta)$ absolutely convergent and bounded on $N_{q}(\delta)$. Then there exists a unique $G \in(\mathcal{S})_{-1}$ such that $\mathcal{H} G=g$, namely

$$
G=\sum_{\alpha \in \mathcal{J}} b_{\alpha} K_{\alpha}
$$

Here is a general solution method for Wick type SPDEs in $(\mathcal{S})^{*}$ or $(\mathcal{S})_{-1}$ :

Theorem 3.19 (9]). Consider a general Wick type SPDE of the form

$$
A^{\diamond}\left(t, x, \partial_{t}, \nabla_{x}, U, \omega\right)=0 \quad \text { in }(\mathcal{S})_{-1} .
$$

Suppose $u(t, x, \zeta)$ is a solution of the Hermite transformed equation

$$
\tilde{A}\left(t, x, \partial_{t}, \nabla_{x}, u, \zeta\right)=0, \quad \zeta \in N_{q}(R)
$$

for $(t, x)$ in some bounded open set $G \subset \mathbb{R} \times \mathbb{R}^{d}$, for some $q, R$. Moreover, suppose that $u(t, x, \zeta)$ and all its partial derivatives involved in (3.46) are uniformly bounded for 
$(t, x, \zeta) \in G \times N_{q}(R)$, continuous with respect to $(t, x) \in G$ for each $\zeta \in N_{q}(R)$ and analytic w.r.t. $\zeta \in N_{q}(R)$ for all $(t, x) \in G$. Then there exists $U(t, x) \in(\mathcal{S})_{-1}$ s.t. $\mathcal{H} U(t, x)=u(t, x, \cdot)$ and $U(t, x)$ solves

$$
A^{\diamond}\left(t, x, \partial_{t}, \nabla_{x}, U, \omega\right)=0 \quad \text { in }(\mathcal{S})_{-1} .
$$

4. Applications. In this section we look at some specific SPDEs driven by Lévy white noise and indicate how the general theory of the previous section can be used to solve them.

EXAMPle 4.1. Temperature distribution in a region with a Lévy white noise heat source.

Consider the SPDE in Example 2.3, i.e.

$$
\begin{aligned}
\Delta U(x) & =-\dot{\eta}(x), & & x \in D, \\
U(x) & =0, & & x \in \partial D,
\end{aligned}
$$

where $\dot{\eta}(x)=m_{2} \sum_{j=1}^{\infty} \xi_{j}(x) K_{\left.\epsilon^{(\kappa(j, 1)}\right)}$ is $d$-parameter (space) white noise (see (3.34) ) and $D \subset \mathbb{R}^{d}$ is a given bounded domain with $C^{1}$ boundary.

We regard this as an equation in $(\mathcal{S})_{-1}$ and we seek a solution $U: \bar{D} \rightarrow(\mathcal{S})_{-1}$ such that (4.1)-(4.2) hold pointwise in $x$.

To solve this equation we consider its $\mathcal{H}$-transform:

$$
\begin{aligned}
& \Delta u(x ; \zeta)=-m_{2} \sum_{j=1}^{\infty} \xi_{j}(x) \zeta_{\kappa(j, 1)}(=-\mathcal{H} \eta(\zeta)), \quad x \in D, \\
& u(x ; \zeta)=0, \quad x \in \partial D
\end{aligned}
$$

where

$$
\zeta=\left(\zeta_{1}, \zeta_{2}, \ldots\right) \in\left(\mathbb{C}^{\mathbb{N}}\right)_{c}
$$

We solve this equation for a given $\zeta \in\left(\mathbb{C}^{\mathbb{N}}\right)_{c}$ as a parameter and get

$$
u(x, \zeta)=m_{2} \sum_{j=1}^{\infty}\left(\int_{D} G(x, y) \xi_{j}(y) d y\right) \zeta_{\kappa(j, 1)}
$$

where $G$ denotes the Green function for the Laplacian in $D$.

One can now verify that $u(x, \zeta)$ satisfies the requirement of Theorem 3.19, and hence that there exists $U(x) \in(\mathcal{S})_{-1}$ such that $\mathcal{H} U(x)=u(x)$ and $U(x)$ solves (4.1)-(4.2).

This is the main idea of the proof of the following result:

Theorem 4.2 (14]). There exists a unique stochastic distribution process $U: \bar{D} \rightarrow(\mathcal{S})^{*}$ satisfying (4.1)-(4.2). The solution is $C^{2}$ in $(\mathcal{S})^{*}$ and has the form

$$
U(x)=\int_{D} G(x, y) \dot{\eta}(y) d y=m_{2} \sum_{j=1}^{\infty}\left(\int_{D} G(x, y) \xi_{j}(y) d y\right) K_{\epsilon^{(\kappa(j, 1))}} .
$$

In some cases the solution can be proved to belong to a smaller space than $(\mathcal{S})^{*}$ : 
Corollary 4.3 ([14]). Suppose $d \leq 3$. Then the solution $U(x) \in L^{2}(P)$ for each $x$ and it is continuous in $x$. Moreover,

$$
U(x)=\int_{D} G(x, y) \delta \eta(y)\left(=\int_{D} G(x, y) d \eta(y)\right) .
$$

For general $d$ we have the following interpretation of our solution $U(x) \in(\mathcal{S})^{*}$ : for each $x, U(x)$ is a stochastic distribution whose action on a stochastic test function $f \in(\mathcal{S})$ is given by

$$
(U(x), f)=\int_{D} G(x, y)(\dot{\eta}(y), f) d y
$$

where

$$
(\dot{\eta}(y), f)=\sum_{j=1}^{\infty} \xi_{j}(y) E\left[K_{\epsilon(\kappa(j, 1))} f\right]
$$

Remark. Equation (4.1)-(4.2) can also be solved using the Fourier series in eigenfunctions of the Dirichlet Laplacian. The result would be the same. In fact, in this case the solution also coincides with the solution one would obtain by using the Walsh method 22 .

EXAMPLE 4.4 (19]). Waves in a region with a Lévy white noise force.

Let $D \subset \mathbb{R}^{m}$ be a bounded domain with a $C^{1}$ boundary. Consider the stochastic wave equation

$$
\begin{aligned}
\frac{\partial^{2} U}{\partial t^{2}}(t, x)-\Delta U(t, x) & =F(t, x) \in C^{\frac{m+1}{2}}\left(\mathbb{R}_{+} \times \mathbb{R}^{m} ;(\mathcal{S})_{-1}\right), \\
U(0, x) & =G(x) \in C^{\frac{m+3}{2}}\left(\mathbb{R}^{m} ;(\mathcal{S})_{-1}\right), \\
\frac{\partial U}{\partial t}(0, x) & =H(x) \in C^{\frac{m+1}{2}}\left(\mathbb{R}^{m} ;(\mathcal{S})_{-1}\right) .
\end{aligned}
$$

Here

$$
\begin{array}{ll}
F(\cdot, \cdot): \mathbb{R}_{+} \times \mathbb{R}^{m} \rightarrow(\mathcal{S})_{-1} & \text { (corresponding to } d=m+1), \\
G(\cdot): \mathbb{R}^{m} \rightarrow(\mathcal{S})_{-1} & \text { (corresponding to } d=m),
\end{array}
$$

and

$$
H(\cdot): \mathbb{R}^{m} \rightarrow(\mathcal{S})_{-1}
$$

are given stochastic distribution processes.

By applying the Hermite transform, then solving the corresponding deterministic PDE for each value of the parameter $\zeta \in\left(\mathbb{C}^{\mathbb{N}}\right)_{c}$ and finally taking the inverse Hermite transform as in the previous example, we get an $(\mathcal{S})_{-1}$-valued solution (in any dimension $m$ ). To illustrate this we just give the solution in the case $m=1$ and we refer to [19] for a solution in the general dimension. 
Theorem 4.5 ([19], $m=1$ case). If $m=1$, then the unique solution $U(t, x)$ of equation (4.10)-(4.12) is

$$
\begin{gathered}
U(t, x)=\frac{1}{2}(G(x+t)-G(x-t))+\frac{1}{2} \int_{x-t}^{x+t} H(s) d s \\
+\frac{1}{2} \int_{0}^{t} \int_{x-(t-s)}^{x+(t-s)} F(s, y) d y d s .
\end{gathered}
$$

Here the integrals are $(\mathcal{S})^{*}$-valued integrals.

In particular, if $F(s, y)=\dot{\eta}(s, y)$, then the last term can be written as

$$
\frac{1}{2} \eta\left(D_{t, x}\right)
$$

where $D_{t, x}=\{(s, y) ; x-t+s \leq y \leq x+t-s, 0 \leq s \leq t\}$ is the domain of dependence of the point $(t, x)$.

EXAMPLE 4.6. Heat propagation in a domain with a Lévy white noise potential.

Consider the stochastic heat equation

$$
\begin{aligned}
& \frac{\partial U}{\partial t}(t, x)=\frac{1}{2} \Delta U(t, x)+U(t, x) \diamond \dot{\eta}(t, x), \quad(t, x) \in[0, T] \times \mathbb{R}^{d}, \\
& U(0, x)=f(x), \quad x \in \mathbb{R}^{d}(f \text { deterministic }) .
\end{aligned}
$$

We take the Hermite transform and get the following deterministic heat equation in $u(t, x ; \zeta)$ with $\zeta \in\left(\mathbb{C}^{\mathbb{N}}\right)_{c}$ as a parameter:

$$
\begin{aligned}
& \frac{\partial}{\partial t} u(t, x ; \zeta)=\frac{1}{2} \Delta u(t, x ; \zeta)+u(t, x ; \zeta) \mathcal{H} \dot{\eta}(t, x ; \zeta), \\
& u(0, x ; \zeta)=f(x) .
\end{aligned}
$$

This equation can be solved by using the Feynman-Kac formula, as follows:

Let $\hat{B}(t)$ be an auxiliary Brownian motion on a filtered probability space $(\hat{\Omega}, \hat{\mathcal{F}}$, $\left.\left\{\mathcal{F}_{t}\right\}_{t \geq 0}, \hat{P}\right)$, independent of $B(\cdot)$. Then the solution of (4.16)-(4.17) can be written

$$
u(t, x ; \zeta)=\hat{E}^{x}\left[f(\hat{B}(t)) \exp \left(\int_{0}^{t} \mathcal{H} \dot{\eta}(s, \hat{B}(s) ; \zeta) d s\right)\right],
$$

where $\hat{E}^{x}$ denotes expectation with respect to $\hat{P}$ when $\hat{B}(0)=x$. Taking inverse Hermite transforms we get:

Theorem 4.7. The unique $(\mathcal{S})_{-1}$-solution of $(4.14)-(4.15)$ is

$$
U(t, x)=\hat{E}^{x}\left[f(\hat{B}(t)) \exp ^{\diamond}\left(\int_{0}^{t} \dot{\eta}(s, \hat{B}(s)) d s\right)\right],
$$

where $\exp ^{\diamond}(\cdot)$ denotes the Wick exponential, defined in general by

$$
\exp ^{\diamond} F=\sum_{n=0}^{\infty} \frac{1}{n !} F^{\diamond n}, \quad F \in(\mathcal{S})_{-1},
$$

where

$$
F^{\diamond n}=F \diamond F \diamond \cdots \diamond F \quad(n \text { times }) .
$$

We refer to [9] for more details. 
Final REMARKs. From the examples above one might get the impression that the white noise theory can only be used to solve the linear SPDEs. This is not the case. In fact, in 13 it is shown how white noise theory (for Brownian motion) can be used to find a remarkable explicit solution formula for the general nonlinear stochastic differential equation

$$
\begin{aligned}
d X(t) & =b(X(t)) d t+\sigma(X(t)) d B(t), \quad 0 \leq t \leq T, \\
X(0) & =x \in \mathbb{R} \quad \text { (fixed), }
\end{aligned}
$$

where $b$ and $\sigma$ are given functions satisfying the usual Lipschitz conditions. They assume that

$$
\sigma(x)>0 \text { for all } x \text { and } \sigma \in C^{1}(\mathbb{R})
$$

and

$$
\frac{b(x)}{\sigma(x)} \text { is bounded on } \mathbb{R} \text {. }
$$

Define

$$
\Lambda(y)=\int_{x}^{y} \frac{1}{\sigma(u)} d u .
$$

Let $\varphi: \mathbb{R} \rightarrow \mathbb{R}$ be a given measurable function and let $\hat{B}$ be as above. Define

$$
Y(s)=\dot{B}(s)-\frac{b\left(\Lambda^{-1}(\hat{B}(s))\right)}{\sigma\left(\Lambda^{-1}(\hat{B}(s))\right)}+\frac{1}{2} \sigma^{-1}\left(\Lambda^{-1}(\hat{B}(s))\right)
$$

and

$$
M_{T}^{\diamond}=\exp ^{\diamond}\left(\int_{0}^{T} Y(s) d \hat{B}(s)-\frac{1}{2} \int_{0}^{T} Y^{\diamond 2}(s) d s\right) .
$$

Here $\dot{B}(s)=\frac{d B(s)}{d s}$ is the white noise in $\left(\mathcal{S}^{\prime}(\mathbb{R}), \mathcal{F}, P\right)$ as before and $\int_{0}^{T} \dot{B}(s) d \hat{B}(s)$ is the $(\mathcal{S})^{*}$-valued stochastic integral with respect to $d \hat{B}(s)$, while $\diamond$ is the Wick product with respect to $B$. Then we have the following amazing result:

Theorem 4.8 (13. (General solution formula for SDEs)). Let $X(t)$ be the unique strong solution of (4.20)-(4.21). Assume that (4.22) and (4.23) hold. Then

$$
\varphi(X(t))=\hat{E}\left[\varphi\left(\Lambda^{-1}(\hat{B}(t))\right) M_{T}^{\diamond}\right],
$$

where $\hat{E}$ denotes expectation with respect to $\hat{P}$.

\section{REFERENCES}

[1] D. Applebaum: Lévy Processes and Stochastic Calculus. Cambridge Univ. Press, 2004. MR.2072890 (2005h:60003)

[2] F. E. Benth: Integrals in the Hida distribution space $(\mathcal{S})^{*}$. In T. Lindstrøm, B. Øksendal and A. S. Üstunel (editors): Stochastic Analysis and Related Topics. Gordon and Breach, 1993, pp. 89-99. MR 1268006 (95e:60052)

[3] F. E. Benth and A. Løkka: Anticipative calculus for Lévy processes and stochastic differential equations. Stochastics and Stochastics Reports 76 (2004), 191-211. MR2072380 (2006g:60081)

[4] R. Cont and P. Tankov: Financial Modelling with Jump Processes. Chapman \& Hall/CRL 2004. MR2042661 (2004m:91004)

[5] G. Di Nunno, B. Øksendal and F. Proske: White noise analysis for Lévy processes. J. Funct. Anal. 206 (2004), 109-148. MR2024348 (2004j:60148)

[6] G. Di Nunno, B. Øksendal and F. Proske: Malliavin Calculus for Lévy Processes and Applications to Finance. Forthcoming book, to be published by Springer. 
[7] T. Hida: Brownian Motion. Springer, 1980. MR562914 (81a:60089)

[8] T. Hida, H.-H. Kuo, J. Potthoff and L. Streit: White Noise. Kluwer, 1993. MR.1244577 (95f:60046)

[9] H. Holden, B. Øksendal, J. Ubøe and T. Zhang: Stochastic Partial Differential Equations. Birkhäuser, 1996. Second Edition to appear in 2008/2009. MR1408433 (98f:60124)

[10] K. Itô: Multiple Wiener Integral. J. Math. Soc. Japan 3 (1951), 157-169. MR0044064 (13:364a)

[11] Y. Kabanov: A generalized Itô formula for an extended stochastic integral with respect to a Poisson random measure. (In Russian). Usp. Mat. Nauk 29 (1974), 167-168. MR0397876 (53:1732)

[12] Y. Kabanov: On extended stochastic integrals. Theory Probab. Appl. 20 (1975), 710-722. MR0397877 (53:1733)

[13] A. Lanconelli and F. Proske: On explicit strong solution of Itô-SDEs and the Donsker delta function of a diffusion. Inf. Dim. Anal. Quant. Probab. 7 (2004), 437-447. MR.2085642 (2005h:60186)

[14] A. Løkka, B. Øksendal and F. Proske: Stochastic partial differential equations driven by Lévy space-time white noise. Annals Appl. Probab. 14 (2004), 1506-1528. MR2071432 (2005d:60078)

[15] T. Lindstrøm, B. Øksendal and J. Ubøe: Wick multiplication and Itô-Skorohod stochastic differential equations. In S. Albeverio et al. (editors): "Ideas and Methods in Mathematical Analysis, Stochastics and Applications". Cambridge Univ. Press, 1992, pp. 183-206. MR.1190500 (94d:60084)

[16] A. Løkka and F. Proske: Infinite dimensional analysis of pure jump Lévy processes on the Poisson space. Math. Scand. 98 (2006), 237-261. MR2243705

[17] D. Nualart and W. Schoutens: Chaotic and predictable representations for Lévy processes. Stoch. Proc. Appl. 90 (2000), 109-122. MR.1787127(2001j:60090)

[18] B. Øksendal and F. Proske: White noise of Poisson random measures. Potential Analysis 21 (2004), 375-403. MR2081145 (2005i:60134)

[19] B. Øksendal, F. Proske and M. Signahl: The Cauchy problem for the wave equation with Lévy noise initial data. Inf. Dim. Anal. Quantum Probab. Rel. Topics 9 (2006) 249-270. MR2235547 (2007e:60059)

[20] B. Øksendal and A. Sulem: Applied Stochastic Control of Jump Diffusions. Springer. Second Edition 2007. MR 2322248

[21] K.-I. Sato: Lévy Processes and Infinitely Divisible Distributions. Cambridge Univ. Press, 1999. MR 1739520 (2003b:60064)

[22] J. B. Walsh: An introduction to stochastic partial differential equations. In R. Carmona, H. Kesten and J. B. Walsh (editors); École d'Été de Probabilités de Saint-Flour XIV-1984. Springer Lecture Notes in Math. 1180, pp. 265-439. MR876085 (88a:60114) 